\title{
High-dimensional exciton-vibrational wave- packet dynamics in the FMO complex. influence of site-specific spectral densities
}

\author{
Jan Schulze ${ }^{1}$, Mohamed F. Shibl ${ }^{2}$, Mohammed J. Al-Marri ${ }^{2}$ and Oliver Kühn ${ }^{1, *}$ \\ ${ }^{1}$ Institut für Physik, Universität Rostock, Albert-Einstein-Straße 23, 18059 Rostock, Germany \\ ${ }^{2}$ Department of Chemistry, College of Engineering, Qatar University, P.O. Box 2713, Doha, Qatar
}

\begin{abstract}
The correlated exciton-vibrational dynamics of the FennaMatthews-Olson (FMO) complex is studied using Multi-layer Multiconfiguration Time-dependent Hartree (ML-MCTDH) wavepacket propagation. Exciton populations and coherences are shown to be sensitive to the details of the spectral density.
\end{abstract}

\section{Introduction}

The FMO pigment-protein complex has taken a prominent role for our understanding of dynamics and spectroscopy of electronic energy transfer in photosynthesis. In particular, two-dimensional (2D) electronic spectroscopy has gathered evidence for long-lived coherences up to physiological temperatures [1], although this result has been challenged recently [2]. Different experimental results are paralleled by a variety of theoretical predictions (e.g. [2-4]). This holds true despite the fact that the underlying model is identical, i.e. the Frenkel exciton Hamiltonian with linear coupling of the local electronic excitations to a bath of harmonic modes of the bacteriochlorophyll monomer as well as of the protein environment. The exciton-vibrational coupling is represented by the spectral density (SD), i.e. the distribution of Huang-Rhys factors with respect to the modes' frequencies [5]. The SD can be extracted from fluorescence excitation spectra [6], but also a number of (different) theoretical SDs yielding different dynamics have been reported (e.g. [7]).

Exciton dynamics in photosynthesis is commonly described using a reduced density matrix system-bath approach [5]. Although the equations of motion can be solved numerically exactly [4], explicit information with respect to the dynamics of vibrational degrees of freedom is not available. Thus, the possible roles of particular modes can be assessed indirectly only. Recently, we have used high-dimensional exciton-vibrational wavepacket propagation to gain detailed insight into the exciton transfer in FMO $[8,9]$. Solving the time-dependent Schrödinger equation in hundreds of dimensions becomes possible by the ML-MCTDH approach (for a review, see [11]). Here, the state vector $|\Psi(\boldsymbol{Q} ; t)\rangle=\sum_{m} \chi_{m}(\boldsymbol{Q} ; t)|m\rangle$ is expanded with respect to the local exciton states, $|m\rangle$, and

\footnotetext{
*Corresponding author: oliver.kuehn@uni-rostock.de
} 
the different wavepackets, $\chi_{m}(\boldsymbol{Q} ; t)$, are expressed in terms of Hartree product configurations of time-dependent basis functions.

Using this approach, two different mechanism have been identified, which lead to mode-specific effects [9]. First, there is a competition between local vibrational motion and exciton transfer, triggering electronic ground state vibrational motion if the vibrational frequency exceeds the transfer coupling. Second, energetic downhill transfer is vibronically assisted, leading to vibronic excitation in a narrow frequency range. Here, we explore this model further, focusing on the effect of the SD model on the population and coherence dynamics.
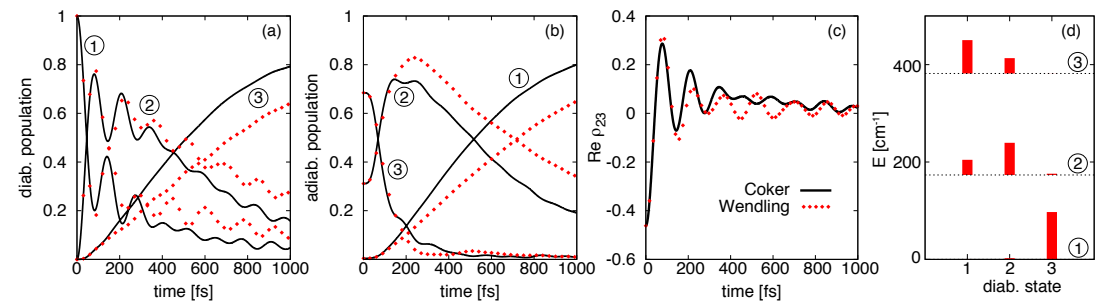

Fig. 1. Population dynamics using the Wendling and Coker SDs ((a) diabatic basis, (b) adiabatic basis, decomposition of adiabatic into diabatic states is given in panel (d)). Panel (c) shows the coherence evolution between adiabatic states 2 and 3 (corresponding to an off-diagonal peak in the 2D spectra) for both SDs. Numbers in circles label the respective states.

\section{Results and Discussion}

Simulation results are shown for the three-site $(m=1-3)$ FMO model as described in [10] using the Heidelberg MCTDH package [11]. Two different SDs will be compared, i.e. the discretized experimental Wendling SD [6], which is uniform for all sites, and the calculated site-specific Coker SD [7]. In the following we will discuss the one-exciton reduced density matrix in diabatic (site) representation, $\rho_{m n}(t)=\langle\Psi(t) \mid m\rangle\langle n \mid \Psi(t)\rangle$, as well as in the adiabatic (eigenstate), $\rho_{\alpha \beta}(t)$, representation. The eigenstates are defined as $|\alpha\rangle=\sum_{m} C_{m}(\alpha)|m\rangle$, the squared coefficients for the used excitonic model Hamiltonian are shown in Fig. 1(d). Apparently, sites 1 and 2 are strongly coupled, whereas site 3 is only weakly mixed with site 2 .

The diabatic population dynamics upon initial excitation of site 1 is shown in Fig. 1(a) for the Wendling and Coker SDs. First, we notice that the overall behavior is rather similar to previous reduced density matrix calculations $[3,4]$, i.e. there is a beating between sites 1 and 2, which is damped on the time scale of about $1 \mathrm{ps}$, along with a decay into site 3 whose dynamics doesn't show any oscillations. We emphasize, however, that the decay is solely an effect of the high-dimensional vibrational space and not the result of phenomenologically introduced rates. The adiabatic population dynamics in Fig. 1(b) doesn't show the rapid oscillations and merely reflects the decay of the initial exciton eigenstate populations. The Wendling and Coker SDs differ in shape as well as in the integrated Huang-Rhys factor (Wendling: 0.42, Coker: $(0.64,0.96,0.58)$ for sites $(1,2,3))$. In [10], it was argued that the total Huang-Rhys factor determines the effective decays rates, whereas vibrational and vibronic excitation are sensitive towards the actual shape of the SD. The dependence of the population and also the coherence decay on the SD model is confirmed by Fig. 1(a-c).

A more global view on the dynamics is provided by the time-averaged exciton density matrix in diabatic representation, $\left\langle\left|\rho_{m n}\right|\right\rangle_{T}=T^{-1} \int_{0}^{T} d t\left|\rho_{m n}(t)\right|$. In Figs. 2(b) and (c), 
Wendling and Coker SD results are compared. Obviously, population trapping at site 3 is more pronounced for the Coker SD, whereas for the Wendling SD, populations stays longer at site 2 . Inspecting coherences, one notices that those between sites 1 and 2 have a larger average for the Wendling SD, whereas the coherence between sites 2 and 3 is essentially not affected by the choice of the SD. Finally, both results can be compared to a bare excitonic model, where excitation is essentially trapped at sites 1 and 2 (Fig. 2(a)).
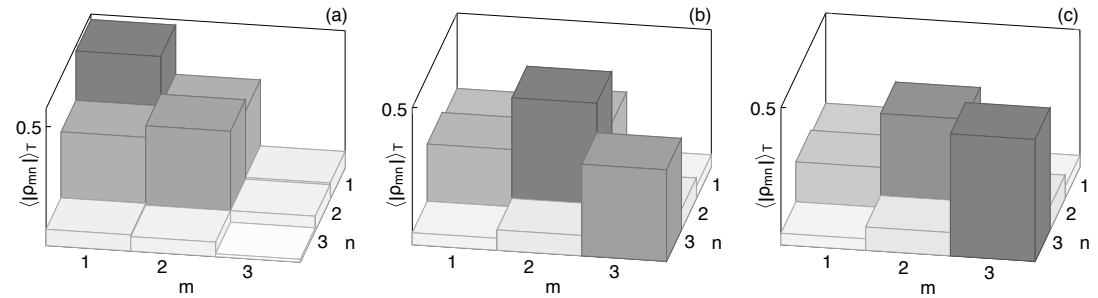

Fig. 2. Time-averaged exciton density matrix elements for the pure excitonic (a), the Wendling SD (b), and the Coker SD (c) models.

\section{Conclusions}

ML-MCTHD high-dimensional exciton-vibrational wavepacket dynamics, combined with well-established model Hamiltonians, provide the means to study excitation energy transfer in photosynthetic systems such as the FMO complex. Compared to reduced density matrix approaches, this provides the advantage of having explicit information with respect to the vibrational degrees of freedom. Further, phase and energy relaxation occur not due to respective rates, but as a consequence of the high dimensionality of the configuration space. Applying this method to the specific case of the FMO complex, we have shown that the actual model for the SD, in fact, matters leading to different dynamics of excitonic populations and coherences.

This work was made possible by NPRP grant \#NPRP 7-2271-034 from the Qatar National Research Fund (a member of Qatar Foundation). The statements made herein are solely the responsibility of the authors.

[1] G. Panitchayangkoon, D. Hayes, K.A. Fransted, J.R. Caram, E. Harel, J. Wen, R.E. Blankenship, G.S. Engel, Proc. Natl. Acad. Sci. 107, 12766 (2010).

[2] H.G. Duan, V. I. Prokhorenko, R. J. Cogdell, K. Ashraf, A.L. Stevens, M. Thorwart, R.J.D. Miller, Proc. Natl. Acad. Sci. 114, 8493 (2017).

[3] A. Ishizaki, T.R. Calhoun, G.S. Schlau-Cohen, G.R. Fleming, PhysChemChemPhys 12, 7319 (2010).

[4] C. Kreisbeck and T. Kramer, J. Phys. Chem. Lett. 3, 2828 (2012).

[5] M. Schröter, S.D. Ivanov, J. Schulze, S.P. Polyutov, Y. Yan, T. Pullerits, O. Kühn, Phys. Rep. 567, 1 (2015).

[6] M. Wendling, T. Pullerits, M.A. Przyjalgowski, S.I.E. Vulto, T.J. Aartsma, R. van Grondelle, H. van Amerongen, J. Phys. Chem. B 104, 5825 (2000).

[7] M.K. Lee and D. F. Coker, J. Phys. Chem. Lett. 7, 3171 (2016).

[8] J. Schulze, O. Kühn, J. Phys. Chem. B 119, 6211 (2015).

[9] J. Schulze, M.F. Shibl, M.J. Al-Marri, O. Kühn, J. Chem. Phys. 144, 185101 (2016).

[10] J. Schulze, M.F. Shibl, M.J. Al-Marri, O. Kühn, Chem. Phys. 497, 10 (2017).

[11] H.-D. Meyer, WIREs Comput. Mol. Sci. 2, 351 (2011). 